\title{
Drug Interrupted
}

National Cancer Institute

\section{Source}

National Cancer Institute. Drug Interrupted. NCI Thesaurus. Code C49501.

An indication that a medication schedule was modified by temporarily terminating a prescribed regimen of medication. 